\title{
Absence of a Magnetic Phase Transition in Heisenberg, Hubbard, and Kondo-lattice (s-f) Films
}

\author{
Axel Gelfert and Wolfgang Nolting \\ Lehrstuhl Festkörpertheorie, Institut für Physik \\ Humboldt-Universität zu Berlin, Invalidenstraße 110 \\ 10115 Berlin, Germany \\ email: gelfert@physik.hu-berlin.de
}

\begin{abstract}
The possibility of a magnetic phase transition in Heisenberg, Hubbard, and s-f (Kondo-lattice) films is investigated. It is shown that, for any finite temperature $(\beta<\infty)$ and any finite number of layers $(d<\infty)$, the magnetization within every layer must vanish. Thus, the Mermin-Wagner theorem is extended to a variety of system geometries. We also comment on the microscopic interpretation of the transition from two to three dimensions in the limit $d, \beta \rightarrow \infty$.
\end{abstract}

\section{Introduction}

In their well-known 1966 paper, Mermin and Wagner[1] proved rigorously that, at any finite temperature, there can be no spontaneous magnetization in the one- and two-dimensional isotropic Heisenberg ferromagnet. They were also able to exclude the possibility of spontaneous sublattice magnetization in the one- and two-dimensional isotropic two-sublattice antiferromagnet. The proof is based on an inequality originally derived by Bogoliubov in a series of papers.[2] At roughly the same time as Mermin and Wagner, Hohenberg showed there could be no finite-temperature phase transition in one- and twodimensional superfluid systems. [3] His proof, too, is based on Bogoliubov's inequality. 
Following these early papers, a lot of research has been carried out in order to extend the Mermin-Wagner theorem to other systems. Wegner (1967) considered a model describing a system with locally interacting itinerant electrons. [4] Walker and Ruijgrok (1968) discussed a band model for interacting electrons in a metal[5]; Ghosh (1971), more specifically, recovered the Mermin-Wagner theorem for the Hubbard narrow-energy-band model. [6] A proof for the s-d interaction model is given by van den Bergh and Vertogen (1974). [7] A paper by Robaszkiewicz and Micnas (1976) extends the Mermin-Wagner result to a general model with localized and itinerant electrons, covering the modified Zener model, the extended Hubbard model, s-d models, and the model for the magnetic metal-insulator transition as particular cases. [8] In addition to extending the Mermin-Wagner theorem to several microscopic many-body models, generalizations to more complicated geometries were also found. E.g., Baryakhtar and Yablonskii (1975) prove the Mermin-Wagner theorem for systems with an arbitrary number of magnetic sublattices. [9] Their proof not only excludes spontaneous sublattice magnetization but also non-collinear magnetic order with an external field being applied. Thorpe (1971) considers the case of ferromagnetism in phenomenological models with double and higher-order exchange terms [10. These results were extended to the multi-sublattice case by Krzemiński (1976). [1] Recently, Matayoshi and Matayoshi (1997) have discussed models with $n$-th nearest neighbour exchange interactions and tried to extend the MerminWagner theorem to anisotropic exchange interactions [12]; their proof, however, requires extremely special conditions on the parameters of the model which appear to be of no physical significance.

The general procedure is essentially the same for most of the above papers: The Bogoliubov inequality is used with suitable operators defined in such a way as to give an upper bound for the desired order parameter, i.e. the (bulk or sublattice) magnetization. In general, one arrives at an upper bound that, in the limit $B_{0} \rightarrow 0$, behaves as $\sim\left(M B_{0}\right)^{1 / 3}$ or $\sim 1 /\left(\ln M B_{0}\right)^{1 / 2}$ thus excluding a finite value of the order parameter in one or two dimensions, resp. ( $M$ denotes the bulk magnetization and $B_{0}$ is the external magnetic field). In three dimensions, no such behaviour is found, i.e. the usual line of reasoning fails and spontaneous magnetization cannot be ruled out. Within the usual scheme following Mermin and Wagner, the dimensionality of a system enters into the calculation only through the volume element when integrating the final version of the Bogoliubov inequality over $k$-space. Thus, a microscopic picture of the transition from two to three dimensions does not emerge. 
Chester et al. (1969) argue 13 that for three-dimensional Bose systems of finite cross section or thickness the results of the generically one or twodimensional case are, in principle, reproduced. Their main line of argument rests on the operators in the Bogoliubov inequality being defined as Fourier transforms in a restricted space $D \times D \times L$ or $D \times L \times L$, resp., where $D$ remains finite in the thermodynamic limit while $L$ goes to infinity. This way, the operators lack the physically intuitive meaning of the usual real-space or $k$-space operators, which, again, means that no sound microscopic interpretation can be assigned to the transition from two to three dimensions. Similar approaches have been used by Fernández (1970) and by Fisher and Jasnow (1971) to discuss systems of distinguishable interacting quantum particles and spin systems, respectively. [14] [15]

Today, as a result of the rapid progress of thin-film technology in recent years, one can prepare and study systems with restricted geometries, such as films, in great detail. Thus, important parameters, such as the Curie temperature, magnetization and susceptibility, can be measured and discussed as functions of the number of layers $d$ in a magnetic film. Experiments indicate that, for real systems, the transition from 2D to 3D behaviour of, for example, the critical exponent $\beta$, occurs within a narrow crossover region of $d$. 16] The critical temperature $T_{c}$ also shows a strong $d$-dependence, with $T_{c}(d)$ quickly approaching the bulk value $T_{c}(\infty)$ as $d$ increases (see [16], [17]).

Taking these observations into account, it appears desirable to improve one's theoretical understanding of how the transition from the monolayer to the bulk occurs. By referring only to the truly microscopic properties of the respective many-body model, it should be possible to study in detail the transition from two to three dimensions. Work in this direction has been done by studying, both analytically and numerically, film systems with symmetry-breaking contributions to the Hamiltonian (for Heisenberg films with single-ion anisotropy see [18]), thus allowing the study of the Curie temperature as a function of $d$ and the anisotropy parameter. With respect to ferromagnetic itinerant-moment films, layer/overlayer geometries have been investigated [19], giving yet another indication of the relevance of many-body methods for film systems.

At a more fundamental level, the validity of exact results, such as the Mermin-Wagner theorem in two dimensions, to thin films may be tested. In this paper, a proof of the Mermin-Wagner theorem for film systems within three main many-body models (Heisenberg, Hubbard, and s-f model) will be given. 
For reasons of simplicity, we shall restrict our attention to film systems composed of $d$ identical layers stacked on top of each other. The calculations can easily be generalized to account for more complicated geometries. One can think of the film geometry as consisting of a two-dimensional Bravais lattice, i.e. the first layer, with $N$ lattice sites and a $d$-atom basis that corresponds to the $d$ layers being stacked up. Every lattice vector then decomposes into

$$
\vec{R}_{i \alpha}=\vec{R}_{i}+\vec{r}_{\alpha}
$$

where $\vec{R}_{i}$ is a vector of the Bravais lattice and $\vec{r}_{\alpha}$ is the basis vector pointing to the $\alpha$-th layer. In all of the following calculations, Greek indices label layers and Roman indices refer to sites of the Bravais lattice. It should be noted that translational invariance can only be assumed within each layer. E.g., the notion of a reciprocal lattice only makes sense when referring to the twodimensional Bravais lattice. A similar caveat applies to Fourier transforms being thought of as connecting real-space quantities with those defined in wave-vector space.

\section{Model Hamiltonians}

In the following we shall discuss the Mermin-Wagner theorem for the Heisenberg model, the Hubbard model, and the s-f model. For film systems one has to distinguish between Bravais lattice indices and layer indices for all site-dependent quantities, such as spin operators $S_{i \alpha}^{(+,-, x, y, z)}$, annihilation and creation operators $c_{i \alpha}^{(+)}$or coupling constants $J_{i j}^{\alpha \beta}$ which depend on two lattice sites. In this notation, the Hamiltonian for Heisenberg films is given by

$$
H=-\sum_{i j \alpha \beta} J_{i j}^{\alpha \beta}\left(S_{i \alpha}^{+} S_{j \beta}^{-}+S_{i \alpha}^{z} S_{j \beta}^{z}\right)-b \sum_{i \alpha} e^{-i \vec{K} \cdot \vec{R}_{i}} S_{i \alpha}^{z}
$$

where the term $b \sum_{i \alpha} e^{-i \vec{K} \cdot \vec{R}_{i}} S_{i \alpha}^{z}$ is due to the interaction with an external magnetic field $b=\frac{g_{J} \mu_{B} B_{0}}{\hbar}$. The interaction with an external magnetic field leads to the magnetization

$$
M=\frac{1}{N d} \frac{g_{J} \mu_{B}}{\hbar} \sum_{i \alpha} e^{-i \vec{K} \cdot \vec{R}_{i}}\left\langle S_{i \alpha}^{z}\right\rangle \equiv \frac{1}{d} \sum_{\alpha} M_{\alpha}
$$

where the phase factor $e^{-i \vec{K} \cdot \vec{R}_{i}}$ accounts for both ferromagnetic and antiferromagnetic ordering, depending on the choice of $\vec{K}$. We also assume the 
coupling constants $J_{i j}^{\alpha \beta}$ to satisfy some general conditions, i.e.

$$
\begin{gathered}
J_{i j}^{\alpha \beta}=J_{j i}^{\beta \alpha} \\
J_{l l}^{\varepsilon \varepsilon}=0
\end{gathered}
$$

and

$$
\frac{1}{N d} \sum_{\gamma \varepsilon} \sum_{m p}\left|J_{p m}^{\varepsilon \gamma}\right| \frac{\left(\vec{R}_{m}-\vec{R}_{p}\right)^{2}}{4} \equiv \tilde{Q}<\infty .
$$

These conditions are very weak considering that for example the exchange integrals $J$ will usually decay exponentially with distance.

As an example for itinerant-electron systems, we shall discuss the Hubbard model

$$
\begin{aligned}
H & =\sum_{i j \alpha \beta \sigma} T_{i j}^{\alpha \beta} c_{i \alpha \sigma}^{+} c_{j \beta \sigma}+\frac{U}{2} \sum_{i \alpha \sigma} n_{i \alpha \sigma} n_{i \alpha-\sigma}-b \sum_{i \alpha} e^{-i \vec{K} \cdot \vec{R}_{i}} \sigma_{i \alpha}^{z} \\
& =\sum_{i j \alpha \beta \sigma} T_{i j}^{\alpha \beta} c_{i \alpha \sigma}^{+} c_{j \beta \sigma}-\frac{2 U}{3 \hbar^{2}} \sum_{i \alpha} \vec{\sigma}_{i \alpha} \cdot \vec{\sigma}_{i \alpha}+\frac{U \hat{N}_{t o t}}{2}-b \sum_{i \alpha} e^{-i \vec{K} \cdot \vec{R}_{i}} \sigma_{i \alpha}^{z}
\end{aligned}
$$

where $T_{i j}^{\alpha \beta}$ describes the hopping of an electron from lattice site $j$ of the $\beta$-th layer to site $i$ of the $\alpha$-th layer, $U$ is the energy associated with having two electrons at the same lattice site, and $\hat{N}_{t o t}=\sum_{i \alpha}\left(n_{i \alpha \uparrow}+n_{i \alpha \downarrow}\right)$. Again, a term corresponding to an external magnetic field is included. $\sigma_{i \alpha}^{z}$ in the Hubbard model is the $z$-component of the spin of the electrons associated with the respective lattice site, i.e.

$$
\sigma_{i \alpha}^{z}=\frac{\hbar}{2}\left(c_{i \alpha \uparrow}^{+} c_{i \alpha \uparrow}-c_{i \alpha \downarrow}^{+} c_{i \alpha \downarrow}\right)
$$

Similar to the Heisenberg case, we require the hopping constants $T_{i j}^{\alpha \beta}$ to satisfy the isotropy conditions $T_{i j}^{\alpha \beta}=T_{j i}^{\beta \alpha}$ as well as to converge upon summation over all lattice sites:

$$
\frac{1}{N d} \sum_{\gamma \beta} \sum_{n k}\left|T_{n k}^{\gamma \beta}\right| \frac{\left(\vec{R}_{n}-\vec{R}_{k}\right)^{2}}{4} \equiv \tilde{q}<\infty .
$$

In the s-f model one deals with two spin sub-systems, $\left\{\vec{S}_{i \alpha}\right\}$ and $\left\{\vec{\sigma}_{i \alpha}\right\}$, the former consisting of localized $f$-electrons, the latter being associated with itinerant $s$-electrons. With $z_{\sigma=\uparrow}= \pm 1$, the s-f Hamiltonian is 


$$
H=\sum_{i j \alpha \beta \sigma} T_{i j}^{\alpha \beta} c_{i \alpha \sigma}^{+} c_{j \beta \sigma}-\frac{J}{2} \sum_{i \alpha \sigma}\left(z_{\sigma} S_{i \alpha}^{z} n_{i \alpha \sigma}+S_{i \alpha}^{\sigma} c_{i \alpha-\sigma}^{+} c_{i \alpha \sigma}\right)+\sum_{i \alpha} e^{-i \vec{K} \cdot \vec{R}_{i}}\left(S_{i \alpha}^{z}+\sigma_{i \alpha}^{z}\right) .
$$

\section{Bogoliubov inequality and choice of opera- tors}

The original Bogoliubov inequality as derived in [2] is

$$
\left|\left\langle[C, A]_{-}\right\rangle\right|^{2} \leq \frac{\beta}{2}\left\langle\left[A, A^{+}\right]_{+}\right\rangle\left\langle\left[[C, H]_{-}, C^{+}\right]_{-}\right\rangle
$$

where $A$ and $C$ are local operators and $\langle\ldots\rangle$ denotes the thermodynamic expectation value. It should be noted that the two factors on the r.h.s. each are, mathematically, upper bounds to a norm and, thus, greater than or equal to zero. In particular, if, for example, the double commutator depends on some parameter $k$, we will always have

$$
\left\langle\left[[C, H]_{-}, C^{+}\right]_{-}\right\rangle(k)+\left\langle\left[[C, H]_{-}, C^{+}\right]_{-}\right\rangle\left(k^{\prime}\right) \geq\left\langle\left[[C, H]_{-}, C^{+}\right]_{-}\right\rangle(k)
$$

For our purposes a slightly modified version of the Bogoliubov inequality shall be used. Dividing both sides by the double commutator and summing over all wave vectors $\vec{k}$ associated with the two-dimensional Bravais lattice, one arrives at

$$
\sum_{\vec{k}} \frac{\left|\left\langle[C, A]_{-}\right\rangle\right|^{2}}{\left\langle\left[[C, H]_{-}, C^{+}\right]_{-}\right\rangle} \leq \frac{\beta}{2} \sum_{\vec{k}}\left\langle\left[A, A^{+}\right]_{+}\right\rangle
$$

The choice of suitable operators $A$ and $C$ is crucial; it determines whether the inequality will be physically meaningful or not. In film systems long-range magnetic order within a given layer is conceivable where the bulk (or even sub-lattice) magnetization of the whole system vanishes, e.g.

$$
\begin{array}{ll}
\ldots & \uparrow \uparrow \uparrow \uparrow \uparrow \uparrow \uparrow \uparrow \uparrow \uparrow \uparrow \uparrow \uparrow \uparrow \uparrow \uparrow \uparrow \ldots \\
\ldots & \uparrow \downarrow \uparrow \downarrow \uparrow \downarrow \uparrow \downarrow \uparrow \downarrow \uparrow \downarrow \uparrow \downarrow \uparrow \downarrow . . . \\
\ldots & \uparrow \downarrow \uparrow \downarrow \uparrow \downarrow \uparrow \downarrow \uparrow \downarrow \uparrow \downarrow \uparrow \downarrow \uparrow \downarrow . . . \\
\ldots & \downarrow \downarrow \downarrow \downarrow \downarrow \downarrow \downarrow \downarrow \downarrow \downarrow \downarrow \downarrow \downarrow \downarrow \downarrow \downarrow . . .
\end{array}
$$


Excluding long-range magnetic order for every layer within a film would, therefore, be a considerably stronger statement. The general idea of our proof is to use the Bogoliubov inequality to find an upper bound for the layer magnetization $M_{\beta}$, i.e.

$$
M_{\beta} \leq f\left(B_{0}, M\right)
$$

where $f$ is a function that approaches zero as $B_{0} \rightarrow 0$ and does not depend on any layer-specific quantities.

This is best achieved by choosing $C$ as a sum $\sum_{\alpha}(\ldots)$ of spin operators and $A$ as a spin operator associated with a specific layer $\beta$, say. This way, the numerator of the l.h.s of the Bogoliubov inequality will be a layer-dependent quantity, while the double commutator in the denominator will be summed over all lattice sites and thus be layer-independent. One may then expect to be able to replace the r.h.s. of the inequality by a (layer-independent) upper bound. More specifically, for the Heisenberg model we set

$$
A_{(\alpha)} \equiv S_{\alpha}^{-}(-\vec{k}-\vec{K})
$$

and

$$
C \equiv \sum_{\beta} C_{\beta} \equiv \sum_{\beta} S_{\beta}^{+}(\vec{k})
$$

where we have used the Fourier transform

$$
S_{\alpha}^{(+,-, x, y, z)}(\vec{k})=\sum_{i} e^{i \vec{k} \cdot \vec{R}_{i}} S_{i \alpha}^{(+,-, x, y, z)}
$$

Note that in $\vec{k}$-space we have $\left(S_{\beta}^{+}(\vec{k})\right)^{+}=S_{\beta}^{-}(-\vec{k})$ due to the definition of the Fourier transform. The operators $S$ in the context of the Heisenberg model are spin operators for which the usual commutation relations hold true, such as, in $\vec{k}$-space,

$$
\left[S_{\alpha}^{+}\left(\vec{k}_{1}\right), S_{\beta}^{-}\left(\vec{k}_{2}\right)\right]_{-}=2 \hbar \delta_{\alpha \beta} S_{\alpha}^{z}\left(\vec{k}_{1}+\vec{k}_{2}\right)
$$

and

$$
\left[S_{\alpha}^{z}\left(\vec{k}_{1}\right), S_{\beta}^{ \pm}\left(\vec{k}_{2}\right)\right]_{-}= \pm \hbar \delta_{\alpha \beta} S_{\alpha}^{ \pm}\left(\vec{k}_{1}+\vec{k}_{2}\right)
$$

In the Hubbard model a similar definition for the operators $A$ and $C$ is used. However, as mentioned above, the operators $S^{(\cdots)}$ now are built up from fermionic creation and annihilation operators, e.g.

$$
\sigma_{\alpha}^{-}\left(\vec{k}_{1}\right)=\hbar c_{\vec{k}_{1} \alpha \downarrow}^{+} c_{\vec{k}_{1} \alpha \uparrow}
$$


and

$$
\sigma_{\beta}^{+}\left(\vec{k}_{2}\right)=\hbar c_{\vec{k}_{2} \uparrow \beta}^{+} c_{\vec{k}_{2} \beta \downarrow}
$$

The operators $A$ and $C$ are, then,

$$
A_{(\alpha)} \equiv \sigma_{\alpha}^{-}(-\vec{k}-\vec{K})=\hbar c_{-\vec{k}-\vec{K}, \alpha \downarrow}^{+} c_{-\vec{k}-\vec{K}, \alpha \uparrow}
$$

and

$$
C \equiv \sum_{\beta} \sigma_{\beta}^{+}(\vec{k})=\hbar \sum_{\beta} c_{\vec{k} \beta \uparrow}^{+} c_{\vec{k} \beta \downarrow}\left(=\sum_{\beta} C_{\beta}\right)
$$

respectively. The commutation relations for spin operators may, in a purely formal sense, be used in this case as well.

In the $s$-f model, one must be careful not to forget that one is dealing with two separate spin sub-systems, one of which can be described by usual spin operators, while the other is associated with itinerant electrons. The two systems are independent from one another in the sense that spin operators and creation or annihilation operators commute:

$$
\left[S_{i \alpha}^{(+,-, x, y, z)}, c_{j \beta \sigma}^{(+)}\right]_{-}=0
$$

and, thus,

$$
\left[S_{\alpha}^{(+,-, x, y, z)}\left(\vec{k}_{1}\right), c_{\vec{k}_{2} \beta \sigma}^{(+)}\right]_{-}=0
$$

With (19),(18) we define as operators $A$ and $C$ in the s-f model

$$
A_{(\gamma)}=S_{\gamma}^{-}(-\vec{k}-\vec{K})+\sigma_{\gamma}^{-}(-\vec{k}-\vec{K})
$$

and

$$
C \equiv \sum_{\beta} C_{\beta}=\sum_{\beta}\left(S_{\beta}^{+}(\vec{k})+\sigma_{\beta}^{+}(\vec{k})\right)
$$

\section{Evaluation of the Bogoliubov inequality}

\subsection{Hamiltonian-independent quantities}

It is obvious from the structure of the Bogoliubov inequality (12) that the numerators on both sides of the inequality are determined entirely by the choice of operators as discussed in the previous section. The details of 
the many-body model enter the calculation only via the double commutator $\left\langle\left[[C, H]_{-}, C^{+}\right]_{-}\right\rangle$. For simplicity, we shall, therefore, start with the quantity

$$
\begin{aligned}
& \left\langle[C, A]_{-}\right\rangle \\
= & \sum_{\beta}\left\langle\left[S_{\beta}^{+}(\vec{k})+\sigma_{\beta}^{+}(\vec{k}), S_{\gamma}^{-}(-\vec{k}-\vec{K})+\sigma_{\gamma}^{-}(-\vec{k}-\vec{K})\right]_{-}\right\rangle \\
= & \sum_{m n \beta} e^{i \vec{k} \cdot \vec{R}_{m}} e^{-i(\vec{k}+\vec{K}) \cdot \vec{R}_{n}}\left\langle\left[S_{m \beta}^{+}+\hbar c_{m \beta \uparrow}^{+} c_{m \beta \downarrow}, S_{n \gamma}^{-}+\hbar c_{n \gamma \downarrow}^{+} c_{n \gamma \uparrow}\right]_{-}\right\rangle
\end{aligned}
$$

for the s-f model. For reasons described above, $S$ and $\sigma$ operators commute, so the commutator can be evaluated directly using the fundamental commutation relations. This leads to

$$
\begin{aligned}
\left\langle[C, A]_{-}\right\rangle & =2 \hbar \sum_{m n \beta} \delta_{m n} \delta_{\beta \gamma} e^{i \vec{k} \vec{R}_{m}} e^{-i(\vec{k}+\vec{K}) \cdot \vec{R}_{n}}\left\langle S_{m \beta}^{z}+\sigma_{m \beta}^{z}\right\rangle \\
& =\frac{2 \hbar^{2} N}{g_{J} \mu_{B}} M_{\gamma}\left(T, B_{0}\right)
\end{aligned}
$$

where we have introduced the layer magnetization

$$
M_{\gamma}\left(T, B_{0}\right)=\frac{1}{N} \frac{g_{J} \mu_{B}}{\hbar} \sum_{n} e^{-i \vec{K} \cdot \vec{R}_{n}}\left\langle S_{n \gamma}^{z}+\sigma_{n \gamma}^{z}\right\rangle
$$

It is obvious that in both the Heisenberg and the Hubbard model the same relation

$$
\left\langle\left[C, A_{(\gamma)}\right]_{-}\right\rangle=\frac{2 \hbar^{2} N}{g_{J} \mu_{B}} M_{\gamma}\left(T, B_{0}\right)
$$

holds, the only difference being the fact that only one spin system contributes to the magnetization.

The r.h.s. of the Bogoliubov inequality is proportional to the anticommutator sum

$$
\sum_{\vec{k}}\left\langle\left[A, A^{+}\right]_{+}\right\rangle
$$

which, for the s-f model, is given by

$$
\sum_{\vec{k}}\left[A, A^{+}\right]_{+}=\sum_{\vec{k} m n} e^{-i(\vec{k}+\vec{K}) \cdot\left(\vec{R}_{m}-\vec{R}_{n}\right)}\left(\left[\left(S_{n \gamma}^{-}+\hbar c_{n \gamma \downarrow}^{+} c_{n \gamma \uparrow}\right),\left(S_{m \gamma}^{+}+\hbar c_{m \gamma \uparrow}^{+} c_{m \gamma \downarrow}\right)\right]_{+}\right)
$$


Summing the exponential over all $\vec{k}$ gives the delta function $N \delta_{m n}$. The mixed commutators involving both spin and creation/annihilation operators in this case do not vanish, since we have the anticommutator rather than the commutator. Thus, we arrive at the expression

$$
\begin{aligned}
\left\langle\sum_{\vec{k}}\left[A, A^{+}\right]_{+}\right\rangle= & N \sum_{n}\left(\left\langle\left[S_{n \gamma}^{-}, S_{n \gamma}^{+}\right]_{+}\right\rangle+2 \hbar\left\langle c_{n \gamma \downarrow}^{+} c_{n \gamma \uparrow} S_{n \gamma}^{+}+c_{n \gamma \uparrow}^{+} c_{n \gamma \downarrow} S_{n \gamma}^{-}\right\rangle\right. \\
& \left.+\hbar^{2}\left\langle c_{n \gamma \downarrow}^{+} c_{n \gamma \uparrow} c_{n \gamma \uparrow}^{+} c_{n \gamma \downarrow}+c_{n \gamma \uparrow}^{+} c_{n \gamma \downarrow} c_{n \gamma \downarrow}^{+} c_{n \gamma \uparrow}\right\rangle\right)
\end{aligned}
$$

Since we are interested in the quantity $\left\langle\sum_{\vec{k}}\left[A, A^{+}\right]_{+}\right\rangle$as an upper bound in the Bogoliubov inequality, it suffices to find upper bounds for the individual expectation values on the r.h.s. For the Heisenberg term we find

$$
\sum_{n}\left\langle\left[S_{n \gamma}^{-}, S_{n \gamma}^{+}\right]_{+}\right\rangle=2 \sum_{n}\left\langle\left(S_{n \gamma}^{x}\right)^{2}+\left(S_{n \gamma}^{y}\right)^{2}\right\rangle \leq 2 \sum_{n}\left\langle\vec{S}_{n \gamma}^{2}\right\rangle=2 \hbar^{2} S(S+1) N
$$

For the Hubbard contribution we have

$$
\begin{aligned}
\sum_{n}\left\langle c_{n \gamma \downarrow}^{+} c_{n \gamma \uparrow} c_{n \gamma \uparrow}^{+} c_{n \gamma \downarrow}+c_{n \gamma \uparrow}^{+} c_{n \gamma \downarrow} c_{n \gamma \downarrow}^{+} c_{n \gamma \uparrow}\right\rangle \leq & \sum_{n}\left\langle c_{n \gamma \downarrow}^{+} c_{n \gamma \downarrow}\left(1-c_{n \gamma \uparrow}^{+} c_{n \gamma \uparrow}\right)\right. \\
& \left.+c_{n \gamma \uparrow}^{+} c_{n \gamma \uparrow}\left(1-c_{n \gamma \downarrow}^{+} c_{n \gamma \downarrow}\right)\right\rangle \\
\leq & \sum_{n}\left(\left\langle n_{n \gamma \downarrow}\right\rangle+\left\langle n_{n \gamma \uparrow}\right\rangle\right) \\
\leq & 2 N
\end{aligned}
$$

For the mixed terms appearing only in the s-f model, an upper bound is given by

$$
\sum_{n \sigma}\left\langle c_{n \gamma \sigma}^{+} c_{n \gamma-\sigma} S_{n \gamma}^{-\sigma}\right\rangle \leq 2(4+2 S(S+1)) N
$$

as shown in appendix A (see eqn. (71)).

Tabulating these results we have

$$
\sum_{\vec{k}}\left\langle\left[A, A^{+}\right]_{+}\right\rangle \leq 2 \hbar^{2} S(S+1) N^{2}
$$


for the Heisenberg model;

$$
\sum_{\vec{k}}\left\langle\left[A, A^{+}\right]_{+}\right\rangle \leq 2 \hbar^{2} N^{2}
$$

for the Hubbard model; and

$$
\sum_{\vec{k}}\left\langle\left[A, A^{+}\right]_{+}\right\rangle \leq N^{2} \hbar^{2}(4 S(S+1)+10)
$$

for the s-f model.

\subsection{The double commutator $\left\langle\left[[C, H]_{-}, C^{+}\right]_{-}\right\rangle$}

In this section, we shall calculate the remaining double commutator $\left\langle\left[[C, H]_{-}, C^{+}\right]_{-}\right\rangle$ for the individual models and give upper bounds for use in the Bogoliubov inequality.

\subsubsection{The Heisenberg case}

The double commutator $\left\langle\left[[C, H]_{-}, C^{+}\right]_{-}\right\rangle$in this case is

$$
\begin{aligned}
\left\langle\left[[C, H]_{-}, C^{+}\right]_{-}\right\rangle & =\sum_{\gamma \varepsilon}\left\langle\left[\left[S_{\gamma}^{+}(\vec{k}), H_{0}+H_{b}\right]_{-}, S_{\varepsilon}^{-}(-\vec{k})\right]_{-}\right\rangle \\
& =\sum_{\gamma \varepsilon} \sum_{m p} e^{-i \vec{k} \cdot\left(\vec{R}_{m}-\vec{R}_{p}\right)}\left\langle\left[\left[S_{m \gamma}^{+}, H\right]_{-}, S_{p \varepsilon}^{-}\right]_{-}\right\rangle
\end{aligned}
$$

The real-space commutator on the r.h.s. can be easily evaluated using the standard commutation relations. Thus, one arrives at

$$
\begin{aligned}
\left\langle\left[[C, H]_{-}, C^{+}\right]_{-}\right\rangle(\vec{k})= & \sum_{\gamma \varepsilon} \sum_{m p} J_{p m}^{\varepsilon \gamma} \hbar^{2}\left(\left(1-e^{-i \vec{k} \cdot\left(\vec{R}_{m}-\vec{R}_{p}\right)}\right) .\right. \\
& \left.\cdot\left\langle 2 S_{p \varepsilon}^{z} S_{m \gamma}^{z}+S_{m \gamma}^{+} S_{p \varepsilon}^{-}\right\rangle\right) \\
& +2 b \hbar^{2} \sum_{\varepsilon} \sum_{m} e^{-i \vec{K} \cdot \vec{R}_{m}}\left\langle S_{m \varepsilon}^{z}\right\rangle
\end{aligned}
$$

To this we add the double commutator $\left\langle\left[[C, H]_{-}, C^{+}\right]_{-}\right\rangle(-\vec{k})$ which, as discussed above, is a positive real number. Replacing the spin operator expec- 
tation values by the upper bound $2 \hbar^{2} S^{2}$, we find, after some minor algebra,

$$
\left\langle\left[[C, H]_{-}, C^{+}\right]_{-}\right\rangle \leq 4 N d \hbar^{2}\left|B_{0} M\left(T, B_{0}\right)\right|+8 \hbar^{4} S^{2} \sum_{\gamma \varepsilon} \sum_{m p}\left|J_{p m}^{\varepsilon \gamma}\right| \frac{\vec{k}^{2} \cdot\left(\vec{R}_{m}-\vec{R}_{p}\right)^{2}}{4}
$$

where we have already used the fact that

$$
1-\cos \left(\vec{k} \cdot\left(\vec{R}_{m}-\vec{R}_{p}\right)\right) \leq \frac{\vec{k}^{2} \cdot\left(\vec{R}_{m}-\vec{R}_{p}\right)^{2}}{4} .
$$

With the above definition of the constant $\tilde{Q}$, we arrive at the final result

$$
\left\langle\left[[C, H]_{-}, C^{+}\right]_{-}\right\rangle \leq 4 N d \hbar^{2}\left(\left|B_{0} M\left(T, B_{0}\right)\right|+2 \hbar^{2} S^{2} \tilde{Q} \vec{k}^{2}\right)
$$

\subsubsection{The Hubbard case}

Again, we need to calculate the full double commutator $\left\langle\left[[C, H]_{-}, C^{+}\right]_{-}\right\rangle$. With the Hubbard Hamiltonian given in eqn. (6), we have

$$
\sum_{\gamma}\left[\sigma_{\gamma}^{+}(\vec{k}), H\right]_{-}=\hbar \sum_{i j \alpha \beta} T_{i j}^{\alpha \beta} c_{i \alpha \uparrow}^{+} c_{j \beta \downarrow}\left(e^{-i \vec{k} \cdot \vec{R}_{i}}-e^{-i \vec{k} \cdot \vec{R}_{j}}\right)+b \hbar \sum_{\alpha} \sigma_{\alpha}^{+}(\vec{k}+\vec{K})
$$

which still needs to be commuted with $\sum_{\varepsilon} \sigma_{\varepsilon}^{-}(-\vec{k})$. Replacing the expectation values by their modulus, one gets the relevant inequality

$$
\begin{aligned}
\left\langle\left[[C, H]_{-}, C^{+}\right]_{-}\right\rangle(\vec{k})= & \sum_{\gamma \varepsilon}\left\langle\left[\left[\sigma_{\gamma}^{+}(\vec{k}), H_{0}+H_{b}\right]_{-}, \sigma_{\varepsilon}^{-}(-\vec{k})\right]_{-}\right\rangle \\
\leq & \hbar^{2} \sum_{i l \alpha \varepsilon} T_{i l}^{\alpha \varepsilon}\left(e^{-i \vec{k} \cdot\left(\vec{R}_{i}-\vec{R}_{l}\right)}-1\right)\left(\left|\left\langle c_{i \alpha \uparrow}^{+} c_{l \varepsilon \uparrow}\right\rangle\right|+\left|\left\langle c_{l \varepsilon \downarrow}^{+} c_{i \alpha \downarrow}\right\rangle\right|\right) \\
& +2 b \hbar^{2} \sum_{\alpha}\left\langle\sigma_{\alpha}^{z}(\vec{K})\right\rangle .
\end{aligned}
$$

For the same reasons as above, we may now add $\left\langle\left[[C, H]_{-}, C^{+}\right]_{-}\right\rangle(-\vec{k})$ to get an upper bound for the 1.h.s. For the expectation values $\left|\left\langle c_{i \alpha \uparrow}^{+} c_{l \varepsilon \uparrow}\right\rangle\right|+$ $\left|\left\langle c_{l \varepsilon \downarrow}^{+} c_{i \alpha \downarrow}\right\rangle\right|$ we find as an upper bound $\left|\left\langle c_{i \alpha \uparrow}^{+} c_{l \varepsilon \uparrow}\right\rangle\right|+\left|\left\langle c_{l \varepsilon \downarrow}^{+} c_{i \alpha \downarrow}\right\rangle\right| \leq 4$ (see appendix A, eqn. (74), for detailed calculation), so in total we may write, using the above notation,

$$
\left\langle\left[[C, H]_{-}, C^{+}\right]_{-}\right\rangle \leq 4 N d \hbar^{2}\left(\left|B_{0} M\left(T, B_{0}\right)\right|+2 \tilde{q} k^{2}\right) .
$$




\subsubsection{The s-f case}

The s-f double commutator

$$
\left\langle\left[[C, H]_{-}, C^{+}\right]_{-}\right\rangle=\sum_{\gamma \varepsilon}\left\langle\left[\left[S_{\gamma}^{+}(\vec{k})+\sigma_{\gamma}^{+}(\vec{k}), H_{0}+H_{b}\right]_{-}, S_{\varepsilon}^{-}(-\vec{k})+\sigma_{\varepsilon}^{-}(-\vec{k})\right]_{-}\right\rangle
$$

may be computed by considering first the field-independent contribution to the Hamiltonian

$$
H_{0}=\sum_{i j \alpha \beta \sigma} T_{i j}^{\alpha \beta} c_{i \alpha \sigma}^{+} c_{j \beta \sigma}-\frac{J}{2} \sum_{i \alpha \sigma}\left(z_{\sigma} S_{i \alpha}^{z} n_{i \alpha \sigma}+S_{i \alpha}^{\sigma} c_{i \alpha-\sigma}^{+} c_{i \alpha \sigma}\right)
$$

and the respective commutators

$$
\begin{gathered}
{\left[\sigma_{m \beta}^{+}, H_{0}\right]_{-}=\sum_{k \gamma} T_{k m}^{\gamma \beta} \hbar\left(c_{m \beta \uparrow}^{+} c_{m \beta \downarrow}-c_{k \gamma \uparrow}^{+} c_{m \beta \downarrow}\right)-J \hbar\left(S_{m \beta}^{+} \sigma_{m \beta}^{z}-2 S_{m \beta}^{z} \sigma_{m \beta}^{+}\right)} \\
{\left[S_{m \beta}^{+}, H_{0}\right]_{-}=J \hbar\left(S_{m \beta}^{+} \sigma_{m \beta}^{z}-2 S_{m \beta}^{z} \sigma_{m \beta}^{+}\right)}
\end{gathered}
$$

We then find (neglecting, for the moment, the external contribution $H_{b}$ )

$$
\begin{aligned}
\left\langle\left[\left[C, H_{0}\right]_{-}, C^{+}\right]_{-}\right\rangle(\vec{k})= & \sum_{k m n} \sum_{\beta \gamma \delta} e^{-i \vec{k} \cdot\left(\vec{R}_{m}-\vec{R}_{n}\right)} T_{k m}^{\gamma \beta} \hbar^{2} \cdot \\
& \cdot\left\langle\left[\left(c_{m \beta \uparrow}^{+} c_{m \beta \downarrow}-c_{k \gamma \uparrow}^{+} c_{m \beta \downarrow}\right),\left(S_{n \delta}^{-}+c_{n \delta \downarrow}^{+} c_{n \delta \uparrow}\right)\right]_{-}\right\rangle \\
= & -\sum_{k n} \sum_{\beta \gamma \delta}\left(1-e^{i \vec{k} \cdot\left(\vec{R}_{n}-\vec{R}_{m}\right)}\right) \cdot \\
& \cdot\left(\delta_{\delta \beta} T_{k n}^{\gamma \beta} c_{n \delta \downarrow}^{+} c_{k \gamma \downarrow}+\delta_{\delta \gamma} T_{n k}^{\gamma \beta} c_{k \beta \uparrow}^{+} c_{n \delta \uparrow}\right) \hbar^{2}
\end{aligned}
$$

Following the usual procedure of adding $\left\langle\left[[C, H]_{-}, C^{+}\right]_{-}\right\rangle(-\vec{k})$ we arrive at the upper bound

$$
\begin{aligned}
\left\langle\left[\left[C, H_{0}\right]_{-}, C^{+}\right]_{-}\right\rangle \leq & 2 \hbar^{2} \sum_{n k \gamma \beta}\left|T_{n k}^{\gamma \beta}\right|\left(1-\cos \left(\vec{k} \cdot\left(\vec{R}_{n}-\vec{R}_{k}\right)\right)\right) . \\
& \cdot\left(\left|\left\langle c_{n \beta \downarrow}^{+} c_{k \gamma \downarrow}\right\rangle\right|+\left|\left\langle c_{n \beta \uparrow}^{+} c_{k \gamma \uparrow}\right\rangle\right|\right) \\
\leq & 2 \hbar^{2} \sum_{n k \gamma \beta}\left|T_{n k}^{\gamma \beta}\right| \vec{k}^{2}\left(\vec{R}_{n}-\vec{R}_{k}\right)^{2} \\
= & 2 N d \tilde{q} \hbar^{2} \vec{k}^{2}
\end{aligned}
$$


where $\left|\left\langle c_{n \beta \downarrow}^{+} c_{k \gamma \downarrow}\right\rangle\right|+\left|\left\langle c_{n \beta \uparrow}^{+} c_{k \gamma \uparrow}\right\rangle\right| \leq 4$ has already been used.

To this preliminary result, we still need to add the double commutator $\left\langle\left[\left[C, H_{b}\right]_{-}, C^{+}\right]_{-}\right\rangle$with the external part $H_{b}$ of the Hamiltonian. Here, too, we need to take into account the presence of two distinct spin systems. Thus, $H_{b}$ is given by

$$
H_{b}=-B_{0} \frac{\mu_{B}}{\hbar} \sum_{i \alpha}\left(g_{J} S_{i \alpha}^{z}+2 \sigma_{i \alpha}^{z}\right) e^{-i \vec{K} \cdot \vec{R}_{i}}
$$

where the usual definitions of the spin operators apply. As in the previous cases, one gets

$$
\left\langle\left[\left[C, H_{b}\right]_{-}, C^{+}\right]_{-}\right\rangle=-2 \hbar^{2} N d B_{0} M\left(T, B_{0}\right)
$$

where, however, the magnetization is now defined as

$$
M\left(T, B_{0}\right)=\frac{1}{N d} \frac{\mu_{B}}{\hbar} \sum_{i \beta} e^{-i \vec{K} \cdot \vec{R}_{i}}\left(\left\langle g_{J} S_{i \beta}^{z}\right\rangle+2\left\langle\sigma_{i \beta}^{z}\right\rangle\right)
$$

Finally, we arrive at

$$
\left\langle\left[[C, H]_{-}, C^{+}\right]_{-}\right\rangle \leq 2 N d \hbar^{2}\left(\left|B_{0} M\left(T, B_{0}\right)\right|+\tilde{q} \vec{k}^{2}\right)
$$

\section{Proving the absence of spontaneous mag- netization}

Within all three models, we have found for the double commutator

$$
\left\langle\left[[C, H]_{-}, C^{+}\right]_{-}\right\rangle \leq \xi_{0}^{2} N d\left(\left|B_{0} M\left(T, B_{0}\right)\right|+\xi_{1} \vec{k}^{2}\right)
$$

where $\xi_{i}$ are constants depending, at most, on fixed parameters of the respective many-body models. We also know that

$$
\left\langle[C, A]_{-}\right\rangle=\xi_{2} N M_{\gamma}\left(T, B_{0}\right)
$$

and

$$
\sum_{\vec{k}}\left\langle\left[A, A^{+}\right]_{+}\right\rangle \leq 2 \xi_{3} N^{2}
$$

We can now give a generally applicable discussion of the layer magnetization. With the Bogoliubov inequality (12), 


$$
\sum_{\vec{k}} \frac{\left|\left\langle[C, A]_{-}\right\rangle\right|^{2}}{\left\langle\left[[C, H]_{-}, C^{+}\right]_{-}\right\rangle} \leq \frac{\beta}{2} \sum_{\vec{k}}\left\langle\left[A, A^{+}\right]_{+}\right\rangle
$$

we find

$$
\sum_{\vec{k}} \frac{\xi_{2}^{2} N^{2} M_{\gamma}^{2}\left(T, B_{0}\right)}{\xi_{0}^{2} N d\left(\left|B_{0} M\left(T, B_{0}\right)\right|+\xi_{1} \vec{k}^{2}\right)} \leq \xi_{3} \beta N^{2}
$$

The 1.h.s. of the inequality can be replaced by an integral, using the formula

$$
\sum_{\vec{k}}=\frac{L^{2}}{(2 \pi)^{2}} \int_{\vec{k}} d^{2} \vec{k}
$$

where $\frac{L^{2}}{(2 \pi)^{2}}$ is the area in two-dimensional $\vec{k}$-space associated with one quantum state. Restricting the support of the integral to a finite-volume sphere inscribed into the first Brillouin zone only strengthens the inequality, so

$$
\left(\frac{\xi_{2}}{\xi_{0}}\right)^{2} \frac{1}{2 \pi d} \frac{L^{2}}{N} M_{\gamma}^{2}\left(T, B_{0}\right) \int_{0}^{k_{0}} d k \frac{k}{\left|B_{0} M\left(T, B_{0}\right)\right|+\xi_{1} k^{2}} \leq \xi_{3} \beta
$$

where $k_{0}$ is the cutoff corresponding to the sphere in $\vec{k}$-space. In the thermodynamic limit, $\frac{L^{2}}{N}=v_{0}^{(2)}$ approaches a constant finite value as $N \rightarrow \infty$. Evaluating the integral and performing some minor algebra, we have

$$
M_{\gamma}^{2}\left(T, B_{0}\right) \leq \xi \frac{\beta d}{\ln \left(1+\frac{\xi_{1} k_{0}^{2}}{\left|B_{0} M\left(T, B_{0}\right)\right|}\right)}
$$

( $\xi$ again is an unsignificant constant).

From this formula, it is obvious that, for any finite temperature $(\beta<\infty)$ and finite thickness $(d<\infty)$, the logarithm in the denominator will diverge in the limit $B_{0} \rightarrow 0$, thus forcing the layer magnetization $M_{\gamma}$ to vanish. One should note that the final result does not depend on the choice of $\vec{K}$ and, thus, excludes both ferromagnetic and antiferromagnetic ordering. This proves the Mermin-Wagner theorem for film systems within the Heisenberg, Hubbard, and the s-f (Kondo-lattice) model. 


\section{Summary}

In this paper, we have been able to prove that the Mermin-Wagner theorem which was originally shown to exclude a magnetic phase transition at finite temperature for one and two-dimensional systems can be extended to Heisenberg, Hubbard, and s-f (Kondo-lattice) films of any finite thickness. While this may be expected from very general considerations, and similar theorems for Bose systems and essentially classical systems were proposed before, a microscopic calculation for many-fermion systems has been lacking so far.

In view of recent progress in the experimental and theoretical study of thin magnetic films, it appears to be worthwhile to reconsider the importance of the Mermin-Wagner theorem. This is particularly the case when the symmetry-breaking is not due to explicitly adding anisotropic contributions to the Hamiltonian, but is instead a secondary result of using numerical approximations that violate the Mermin-Wagner theorem. Future work in this direction might be able to provide useful insights for theorists and experimentalists alike.

Our microscopic approach suggests that the parameter $d$, i.e. the number of layers in a film, plays a similar role as the inverse temperature $\beta$. If either one diverges, a phase transition cannot be ruled out. It is, of course, not a priori clear if the behaviour of an upper bound on a physical quantity has any physical significance itself; however, we believe our results to indicate that, within the discussed models, in order to possibly describe (anti)ferromagnetism, one has to take the thermodynamic limit seriously, in the sense that $N \rightarrow \infty$ does not suffice, but rather $d \rightarrow \infty$ is required as well.

\section{Acknowledgments}

Fruitful discussions with Dr. Michael Potthoff are gratefully acknowledged. This work was supported by the Deutsche Forschungsgemeinschaft (DFG) within the SFB 290.

\section{References}

[1] Mermin, N.D. and H. Wagner: Phys. Rev. Lett. 17 (1966) 1133 
[2] Bogoliubov, N.N.: Phys. Abhandl. Sowjetunion 6 (1962), 1, 113, 229

[3] Hohenberg, P.C.: Phys. Rev. 158 (1967) 383

[4] Wegner, F.: Phys. Lett. 24A (1967) 131

[5] Walker, M.B. and T.W. Ruijgrok: Phys. Rev. 171 (1968) 170

[6] Ghosh, D.K.: Phys. Rev. Lett. 27 (1971) 1584

[7] van den Bergh, M. and G. Vertogen: Phys. Lett. A 50 (1974) 85

[8] Robaszkiewicz, S. and R. Micnas: phys. stat. sol. (b) 73 (1976) K35

[9] Baryakhtar, V.G. and D.A. Yablonskii: phys. stat. sol. (b) 72 (1975) K95

[10] Thorpe, M.F.: J. appl. Phys. 42 (1971) 1410

[11] Krzemiński, S.: phys. stat. sol. (b) 74 (1976) K119

[12] Matayoshi, G. and S. Matayoshi: Bull. Coll. Sci. (Univ. Ryukyus) 64 (1997) 1

[13] Chester, G.V.; Fisher, M.E.; and N.D. Mermin: Phys. Rev. 185 (1969) 760

[14] Fernández, J.F.: Phys. Rev. A 2 (1970) 2555

[15] Fisher, M.D. and D. Jasnow: Phys. Rev. B 3 (1971) 907

[16] Li, Y. and K. Baberschke: Phys. Rev. Lett. 68 (1992) 1208

[17] Farle, M.; Baberschke, K.; Stetter, U.; Aspelmeier, A.; and F. Gerhardter: Phys. Rev. B 47 (1993) 11572

[18] Schiller, R. and W. Nolting: Sol. St. Commun. 110 (1999) 121

[19] Wu, J.H.; Herrmann, T.; and W. Nolting: Phys. Rev. B (in press) 


\section{A Upper bound for $\Sigma_{\sigma}\left\langle c_{n \gamma \sigma}^{+} c_{n \gamma-\sigma} S_{n \gamma}^{-\sigma}\right\rangle$ and $\left|\left\langle c_{n \beta \sigma}^{+} c_{k \gamma \sigma}\right\rangle\right|$}

We first discuss the quantity $\sum_{\sigma}\left\langle c_{n \gamma \sigma}^{+} c_{n \gamma-\sigma} S_{n \gamma}^{-\sigma}\right\rangle$. The problem in evaluating the expectation value arises from the fact, that we are dealing with seemingly unrelated operators. It is, therefore, convenient to use the identity

$$
\begin{aligned}
c_{n \gamma \sigma}^{+} c_{n \gamma-\sigma} S_{n \gamma}^{-\sigma}= & \frac{1}{4}\left\{\left(c_{n \gamma \sigma}^{+} c_{n \gamma-\sigma}+S_{n \gamma}^{\sigma}\right)\left(c_{n \gamma-\sigma}^{+} c_{n \gamma \sigma}+S_{n \gamma}^{-\sigma}\right)\right. \\
& -\left(c_{n \gamma \sigma}^{+} c_{n \gamma-\sigma}-S_{n \gamma}^{\sigma}\right)\left(c_{n \gamma-\sigma}^{+} c_{n \gamma \sigma}-S_{n \gamma}^{-\sigma}\right) \\
& +i\left(c_{n \gamma \sigma}^{+} c_{n \gamma-\sigma}+i S_{n \gamma}^{\sigma}\right)\left(c_{n \gamma-\sigma}^{+} c_{n \gamma \sigma}-i S_{n \gamma}^{-\sigma}\right) \\
& \left.-i\left(c_{n \gamma \sigma}^{+} c_{n \gamma-\sigma}-i S_{n \gamma}^{\sigma}\right)\left(c_{n \gamma-\sigma}^{+} c_{n \gamma \sigma}+i S_{n \gamma}^{-\sigma}\right)\right\} \\
\equiv & \frac{1}{4} \sum_{j=1}^{4} \phi(j) B_{j \sigma} B_{j \sigma}^{+}
\end{aligned}
$$

where $j$ labels the individual terms and $\phi$ is a phase factor $(\phi(1)=+1$, $\phi(2)=-1, \phi(3)=i, \phi(4)=-i)$. The original expectation value has thus been decomposed into the sum of expectation values of pairs of adjunct operators $B_{j \sigma} B_{j \sigma}^{+}$. Using the spectral theorem, we find

$$
\sum_{\sigma}\left\langle c_{n \gamma \sigma}^{+} c_{n \gamma-\sigma} S_{n \gamma}^{-\sigma}\right\rangle=\frac{1}{4 \hbar} \sum_{j=1}^{4} \int_{-\infty}^{\infty} d E \frac{1}{e^{\beta E}+1} \phi(j) \sum_{\sigma} S_{B_{j \sigma}^{+} B_{j \sigma}}^{(-)}(E)
$$

where $S_{B_{j \sigma}^{+} B_{j \sigma}}^{(-)}(E)=\frac{1}{2 \pi}\left\langle\left[B_{j \sigma}^{+}, B_{j \sigma}\right]_{+}\right\rangle(E)$ is the spectral density in its energy representation. The l.h.s. is the expectation value of the sum of two adjunct operators and, thus, is real. We may now make use of the fact that for pairs of adjunct operators, the spectral density is positive definite, which, together with the triangle inequality and $|\phi(j)|=1$ gives an upper bound for the r.h.s. :

$$
\frac{1}{4 \hbar} \sum_{j=1}^{4} \int_{-\infty}^{\infty} d E \frac{1}{e^{\beta E}+1} \phi(j) \sum_{\sigma} S_{B_{j \sigma}^{+} B_{j \sigma}}^{(-)}(E) \leq \frac{1}{4} \sum_{\sigma} \sum_{j=1}^{4} \frac{1}{\hbar} \int_{-\infty}^{\infty} d E S_{B_{j \sigma}^{+} B_{j \sigma}}^{(-)}(E)
$$

The sum on the r.h.s. now consists of the 0-th spectral moments associated with the operator pairs $B_{j \sigma}^{+}, B_{j \sigma}$. Each of the spectral moments is given by 
the expectation value of the anticommutator

$$
\frac{1}{\hbar} \int_{-\infty}^{\infty} d E S_{B_{j \sigma}^{+} B_{j \sigma}}^{(-)}(E) \equiv M_{B_{j \sigma}^{+} B_{j \sigma}}^{(0)}=\left\langle\left[B_{j \sigma}^{+}, B_{j \sigma}\right]_{+}\right\rangle .
$$

The anticommutators can be easily evaluated:

$$
\begin{aligned}
M_{B_{1 \sigma}^{+} B_{1 \sigma}}^{(0)}= & \left\langle\left[c_{n \gamma-\sigma}^{+} c_{n \gamma \sigma}+S_{n \gamma}^{-\sigma}, c_{n \gamma \sigma}^{+} c_{n \gamma-\sigma}+S_{n \gamma}^{\sigma}\right]_{+}\right\rangle \\
\leq & \left\langle n_{n \gamma \sigma}-2 n_{n \gamma \sigma} n_{n \gamma-\sigma}+n_{n \gamma-\sigma}\right\rangle+2 S(S+1) \\
& +2\left\langle S_{n \gamma}^{\sigma} c_{n \gamma-\sigma}^{+} c_{n \gamma \sigma}+S_{n \gamma}^{-\sigma} c_{n \gamma \sigma}^{+} c_{n \gamma-\sigma}\right\rangle \\
\leq & 4+2 S(S+1)+2\left\langle S_{n \gamma}^{\sigma} c_{n \gamma-\sigma}^{+} c_{n \gamma \sigma}+S_{n \gamma}^{-\sigma} c_{n \gamma \sigma}^{+} c_{n \gamma-\sigma}\right\rangle \\
\leq & 4+2 S(S+1)-2\left\langle S_{n \gamma}^{\sigma} c_{n \gamma-\sigma}^{+} c_{n \gamma \sigma}+S_{n \gamma}^{-\sigma} c_{n \gamma \sigma}^{+} c_{n \gamma-\sigma}\right\rangle \\
M_{B_{2 \sigma}^{+} B_{2 \sigma}}^{(0)} \leq & 4+2 S(S+1)+2 i\left\langle S_{n \gamma}^{\sigma} c_{n \gamma-\sigma}^{+} c_{n \gamma \sigma}-S_{n \gamma}^{-\sigma} c_{n \gamma \sigma}^{+} c_{n \gamma-\sigma}\right\rangle \\
M_{B_{3 \sigma}^{+} B_{3 \sigma}}^{(0)} \leq & 4+2 S(S+1)-2 i\left\langle S_{n \gamma}^{\sigma} c_{n \gamma-\sigma}^{+} c_{n \gamma \sigma}-S_{n \gamma}^{-\sigma} c_{n \gamma \sigma}^{+} c_{n \gamma-\sigma}\right\rangle \\
M_{B_{4 \sigma}^{+} B_{4 \sigma}}^{(0)} \leq &
\end{aligned}
$$

and hence

$$
\sum_{\sigma}\left\langle c_{n \gamma \sigma}^{+} c_{n \gamma-\sigma} S_{n \gamma}^{-\sigma}\right\rangle \leq 2(4+2 S(S+1))
$$

For the expectation value $\left|\left\langle c_{n \beta \sigma}^{+} c_{k \gamma \sigma}\right\rangle\right|$ one uses the same procedure. For $(n, \beta)=(k, \gamma)$ it is obvious that $\left\langle c_{n \beta \sigma}^{+} c_{n \beta \sigma}\right\rangle \leq 1$. Starting with the decomposition

$$
\begin{aligned}
c_{i \alpha \sigma}^{+} c_{l \nu \sigma}= & \frac{1}{4}\left\{\left(c_{i \alpha \sigma}^{+}+c_{l \nu \sigma}^{+}\right)\left(c_{i \alpha \sigma}+c_{l \nu \sigma}\right)\right. \\
& -\left(c_{i \alpha \sigma}^{+}-c_{l \nu \sigma}^{+}\right)\left(c_{i \alpha \sigma}-c_{l \nu \sigma}\right) \\
& +i\left(c_{i \alpha \sigma}^{+}+i c_{l \nu \sigma}^{+}\right)\left(c_{i \alpha \sigma}-i c_{l \nu \sigma}\right) \\
& \left.-i\left(c_{i \alpha \sigma}^{+}-i c_{l \nu \sigma}^{+}\right)\left(c_{i \alpha \sigma}+i c_{l \nu \sigma}\right)\right\}
\end{aligned}
$$

one can write the expectation value as

$$
\begin{aligned}
\left|\left\langle c_{n \beta \sigma}^{+} c_{k \gamma \sigma}\right\rangle\right| & =\frac{1}{4} \sum_{j=1}^{4} \frac{1}{\hbar} \int_{-\infty}^{\infty} d E|\phi(j)| \frac{1}{e^{\beta E}+1} S_{A_{j} A_{j}^{+}}^{(-)}(E) \\
& \leq \frac{1}{4} \sum_{j=1}^{4} M_{A_{j} A_{j}^{+}}^{(0)}
\end{aligned}
$$


(similar notation as above).

Since we have discussed the case $(n, \beta)=(k, \gamma)$ separately, we can now assume $(n, \beta) \neq(k, \gamma)$. The 0 -th spectral moments can be easily calculated; they each give $M_{A_{j} A_{j}^{+}}^{(0)}=2$. Thus for all $n, k, \beta, \gamma$ we have as an upper bound

$$
\left|\left\langle c_{n \beta \sigma}^{+} c_{k \gamma \sigma}\right\rangle\right| \leq 2
$$

